\title{
EDITORIAL
}

\section{Lactate kinetics in critically ill: a new prognostic marker or just another brick in the wall?}

\author{
Guillaume Geri ${ }^{1,2,3}$, Glenn Hernandez ${ }^{4}$ and Antoine Vieillard-Baron ${ }^{1,2,3^{*}}$
}

(C) 2018 Springer-Verlag GmbH Germany, part of Springer Nature

In this issue of the journal, Masyuk et al. publish another piece of the lactate story [1]. The Surviving Sepsis Campaign has suggested to use lactate normalization as a goal for septic shock resuscitation in patients with hyperlactatemia, although with a low quality of supporting evidence [2]. Indeed, besides hypoperfusion, other sources such as stress-related hyperlactatemia might contribute to persistent hyperlactatemia [3]. Moreover, conflicting results have been reported so far about the efficacy of resuscitation strategies based on serial blood lactate assessments and/or lactate kinetics. One non-inferiority randomized controlled trial (RCT) showed no difference of mortality in 300 septic patients when compared to a strategy based on $\mathrm{ScVO}_{2}$ [4], while another one including 348 unselected patients with an admission blood lactate $\geq 3 \mathrm{mmol} / \mathrm{L}$ reported a beneficial effect on in-hospital mortality, specifically in septic patients [5]. Oddly, in the latter study the authors did not report any lactate kinetics difference during the first $72 \mathrm{~h}$ between control and interventional arms despite an "aggressive" approach in the interventional group [5].

This is in this context that Masyuk et al. report their study [1]. Their primary goal was to investigate prognostic impact of lactate kinetics in a large, real-word cohort of unselected critically ill patients. Among the 6449 patients admitted to the ICU during a 6-year period, they retrospectively analyzed 2191 patients with a lactate blood level $>2 \mathrm{mmol} / \mathrm{L}$ at admission and at least a dosage

\footnotetext{
*Correspondence: antoine.vieillard-baron@aphp.fr

${ }^{1}$ Intensive Care Unit, Section Thorax-Vascular

Disease-Abdomen-Metabolism, Assistance Publique-Hôpitaux de Paris, University Hospital Ambroise Paré, 92100 Boulogne-Billancourt, France

Full author information is available at the end of the article
}

per day [1]. They then calculated the difference between the maximum lactate level at day 1 and at day $2(\Delta 24 \mathrm{Lac})$. $\Delta 24$ Lac was expressed as a percentage when divided by the value at day 1 . Their main results may be summarized as follows. Patients who were not included because of lactate levels at admission below $2 \mathrm{mmol} / \mathrm{L}$ represented $58 \%$ of all admissions and had much lower in-ICU mortality (6\%) as expected. Using ROC curve analysis, Masyuk et al. found that an optimal $\triangle 24 \mathrm{Lac}$ threshold of $19 \%$ best predicted in-ICU mortality ( $43 \%$ and $15 \%$ for patients with a value $\Delta 24 \mathrm{Lac} \leq 19 \%$ and $>19 \%$, respectively). A very surprising result is that $\Delta 24 \mathrm{Lac} \leq 19 \%$ was also associated with long-term mortality (at least 9 years). The authors did not provide any hypothesis about this. We may speculate that long-term mortality difference may indicate that the greater baseline illness severity observed in patients with $\Delta 24 \mathrm{Lac} \leq 19 \%$ was attributable, at least in part, to chronic comorbidities. Indeed, while lactate at admission was not different between the two groups $(5.25 \pm 0.18$ versus $5.10 \pm 0.12 \mathrm{mmol} / \mathrm{L})$, patients with $\triangle 24$ Lac $\leq 19 \%$ were clearly more severely ill with higher SAPS II and APACHE II scores, and intubation rates (56\% versus $42 \%$ ). However, after adjustment based on severity scores as well as the needs for catecholamines and intubation, $\Delta 24 \mathrm{Lac}$ remained significantly associated with mortality.

The design of the study, i.e., purely observational with retrospective analysis, leads to several limitations. Little information regarding hemodynamic monitoring and management is provided, also because of the heterogeneity of the included population. Only $26 \%$ of patients were admitted for sepsis, the main other diagnoses being pneumonia (20\%), acute heart failure (19\%), acute myocardial infarction (29\%), or even pulmonary embolism

\section{Springer}


(3\%). It is also not possible to establish if the different kinetic profiles of lactate were related to increased production (either anaerobic or adrenergic-related aerobic production) or impairment in lactate metabolism due to liver dysfunction, but it should be noted that patients with $\Delta 24$ Lac $\leq 19 \%$ had a significant augmentation in liver enzymes. Moreover, despite a large amount of serial lactate assessments (on average 17 dosages per patient during the first $24 \mathrm{~h}$ leading to 26,285 lactate dosages!), the authors probably missed the opportunity to give us a better picture of lactate changes and mortality, never done before, while it was already reported that lactate kinetics is associated with prognosis [6]. The $\Delta$ Lac24 provided in the paper gives us a truncated and approximate idea of what the modifications of lactate level over time really are at the individual level. Indeed, such an estimate does not take into account (1) the time interval between the two measurements used in the difference and (2) the potential variations of lactate a patient could evidence within the first $24 \mathrm{~h}$. It makes the hypothesis that all the patients have a linear monotonic evolution, missing for instance patients with a U-shaped, J-shaped, or reverse U-shaped evolution of lactate level. This could have been addressed using a mixed-effects model approach. Such a model holds with repeated measurement, taking into account the intra-individual correlation (i.e., the fact that the lactate value at a specific time is strongly associated with subsequent measurements in a particular patient) and can address the issue of different time intervals between assessments as can be observed in non-protocolized data collection [7]. Looking at individual trends on such a large sample study might have provided very original and new insights into a very complicated topic, maybe by discriminating patients according to the shape of lactate evolution within the first $24 \mathrm{~h}$ of resuscitation instead of giving the reader a threshold that might not fit to all.

In conclusion, despite its limitations, the study by Masyuk et al. identifies a cutoff value of 19\% lactate changes to discriminate patients at high risk of death that could be integrated in future studies that will evaluate the clinical effectiveness of lactate-guided resuscitation protocols.

\section{Author details}

${ }^{1}$ Intensive Care Unit, Section Thorax-Vascular Disease-Abdomen-Metabolism, Assistance Publique-Hôpitaux de Paris, University Hospital Ambroise Paré, 92100 Boulogne-Billancourt, France. ${ }^{2}$ Faculty of Medicine Paris lle-de-France Ouest, University of Versailles Saint-Quentin en Yvelines, 78280 Saint-Quentin en Yvelines, France. ${ }^{3}$ INSERM U-1018, CESP, Team 5 (EpReC, Renal and Cardiovascular Epidemiology), UVSQ, 94807 Villejuif, France. ${ }^{4}$ Departamento de Medicina Intensiva, Facultad de Medicina, Pontificia Universidad Católica de Chile, 8331150 Santiago, Chile.

\section{Compliance with ethical standards}

\section{Conflicts of interest}

Guillaume Geri, Glenn Hernandez, and Antoine Vieillard-Baron declare no conflicts of interest.

\section{Publisher's Note}

Springer Nature remains neutral with regard to jurisdictional claims in published maps and institutional affiliations.

Received: 20 November 2018 Accepted: 12 December 2018 Published online: 19 December 2018

References

1. Masyuk M, Wernly B, Lichtenauer M, Franz M, Kabich B, Muessig JM, Zimmermann G, Lauten A, Schulze C, Hoppe UC, Kelm M, Bakker J, Jung C (2018) Prognostic relevance of serum lactate kinetics in critically ill patients. Intensive Care Med. https://doi.org/10.1007/s00134-018-5475-3

2. Rhodes A, Evans LE, Alhazzani W et al (2017) Surviving Sepsis Campaign: international guidelines for management of sepsis and septic shock: 2016. Intensive Care Med 43:304377

3. Hernandez G, Bellomo R, Bakker J (2018) The ten pitfalls of lactate clearance in sepsis. Intensive Care Med. https://doi.org/10.1007/s0013 4-018-5213-x

4. Jones AE, Shapiro NI, Trzeciak S, Arnold RC, Claremont HA, Kline JA, Emergency Medicine Shock Research Network (EMShockNet) Investigators (2010) Lactate clearance vs central venous oxygen saturation as goals of early sepsis therapy: a randomized clinical trial. JAMA 303:739-746

5. Jansen TC, van Bommel J, Schoonderbeek FJ, Sleeswijk Visser SJ, van der Klooster JM, Lima AP, Willemnsen SP, Bakker J, LACTATE study group (2010) Early lactate-guided therapy in intensive care unit patients: a multicenter, open-label, randomized controlled trial. Am J Respir Crit Care Med 182:752-761

6. Vincent JL, Quintairos EA, Couto L, Taccone FS (2016) The value of blood lactate kinetics in critically ill patients: a systematic review. Crit Care 20:257

7. Cnaan A, Laird NM, Slasor P (1997) Using the general linear mixed model to analyse unbalanced repeated measures and longitudinal data. Stat Med 16:2349-2380 